PROCEEDINGS OF THE

AMERICAN MATHEMATICAL SOCIETY

Volume 130, Number 12, Pages 3447-3452

S 0002-9939(02)06791-6

Article electronically published on July 15, 2002

\title{
DISTINCT GAPS BETWEEN FRACTIONAL PARTS OF SEQUENCES
}

\author{
MARIAN VÂJÂITU AND ALEXANDRU ZAHARESCU
}

(Communicated by David E. Rohrlich)

\begin{abstract}
Let $\alpha$ be a real number, $N$ a positive integer and $\mathcal{N}$ a subset of $\{0,1,2, \ldots, N\}$. We give an upper bound for the number of distinct lengths of gaps between the fractional parts $\{n \alpha\}, n \in \mathcal{N}$.
\end{abstract}

\section{INTRODUCTION}

Questions on the distribution of fractional parts of sequences have a long history, and among the most intensively studied are those related to polynomial sequences. After the classical work of Weyl 11 on uniform distribution mod 1, other aspects of the distribution of fractional parts of polynomials, especially questions concerned with small fractional parts, have been investigated (see Schmidt [8] and Baker [1]). Recently, the distribution of gaps between fractional parts of sequences has attracted attention. Following the work of Rudnick and Sarnak [5] on the pair correlation of fractional parts of polynomials, other related questions have been studied in [2], 6] and [7]. We mention that the distribution of the local spacings between the fractional parts $\left\{n^{d} \alpha\right\}, n \in \mathbb{N}$, in the case $d=1$ is completely different than in the case $d>1$. If $d>1$ one expects that for almost all $\alpha$ the distribution is Poissonian, and one knows for instance that the pair correlation is Poissonian indeed (see [5]). If $d=1$ one knows for a fact that the distribution is not Poissonian, and this is a consequence of the following Three Gap Theorem of Steinhaus (see [4], [9] and [10]):

Let $\alpha$ be a real number and $N$ a nonnegative integer. Then the fractional parts $\{n \alpha\}, 0 \leq n \leq N$, partition the unit interval into $N+1$ intervals which have at most 3 different lengths.

The correlation of fractional parts $\{n \alpha\}, n \in \mathbb{N}$, have been recently investigated by Marklof [3. In this paper we take a real number $\alpha$, a positive integer $N$, a subset $\mathcal{N}$ of $\{0,1,2, \ldots, N\}$ and look at the set of fractional parts

$$
\mathcal{M}=\mathcal{M}(\alpha, \mathcal{N})=\{\{n \alpha\}: n \in \mathcal{N}\},
$$

with the intention of proving a result which is independent of $\mathcal{N}$. Clearly, as far as uniform distribution or small fractional parts are concerned, no such result is possible (for instance $\mathcal{N}$ might coincide with the set of those $1 \leq n \leq N$ for which $\left.\{n \alpha\} \in\left[\frac{1}{3}, \frac{1}{2}\right]\right)$. The same goes for the spacing distribution: if $\alpha$ is irrational, then the set $\{n \alpha\}, n \in \mathbb{N}$, is dense in $[0,1]$, and one can choose for large $N$ a sparse

Received by the editors February 7, 2001.

2000 Mathematics Subject Classification. Primary 11K06, 11B05. 
set $\mathcal{N}$ for which the distribution of $\mathcal{M}$ in $[0,1]$ approaches any given distribution. What we will do is to look at the gaps between the elements of $\mathcal{M}$ and see whether any kind of Steinhaus phenomenon still holds in this generality. Thus we arrange the elements of $\mathcal{M}$ in ascending order and consider the number $l(\alpha, \mathcal{N})$ of distinct lengths of gaps between consecutive elements of $\mathcal{M}(\alpha, \mathcal{N})$. Hence $l(\alpha, \mathcal{N}) \leq 3$ when $\mathcal{N}=\{0,1,2, \ldots, N\}$, by the Three Gap Theorem. For a general subset $\mathcal{N}$ of $\{0,1,2, \ldots, N\}, l(\alpha, \mathcal{N})$ can be much larger. For example, if $N$ is a positive integer, $0<\alpha<\frac{1}{N}$ and $\mathcal{N}$ consists of the squares $\left\{0,1,4,9, \ldots,[\sqrt{N}]^{2}\right\}$, then the numbers $n \alpha, n \in \mathcal{N}$, coincide with their fractional parts, and all the gaps between consecutive elements of $\mathcal{M}$ have distinct lengths. Thus $l(\alpha, \mathcal{N})$ can be as large as $\sqrt{N}$. The object of this paper is to prove the following theorem, which shows that $l(\alpha, \mathcal{N})$ cannot be much larger than $\sqrt{N}$.

Theorem 1. For any real number $\alpha$, any positive integer $N$ and any subset $\mathcal{N}$ of $\{0,1,2, \ldots, N\}$ one has

$$
l(\alpha, \mathcal{N})<(2+2 \sqrt{2}) \sqrt{N} .
$$

\section{Proof of Theorem 1}

Fix a positive integer $N$, then choose a real number $\alpha$ and a subset $\mathcal{N}$ of $\{0,1,2, \ldots, N\}$ such that $l(\alpha, \mathcal{N})$ is largest. Note first that for fixed $\mathcal{N}$, the function $\alpha \mapsto l(\alpha, \mathcal{N})$ is periodic mod 1; thus we may assume in what follows that $0 \leq \alpha<1$. In case $\alpha=0$ all the fractional parts $\{n \alpha\}$ are zero, so the maximum value of $l(\alpha, \mathcal{N})$ is attained for some $\alpha \in(0,1)$. Next, notice that for $\mathcal{N}$ fixed, the lengths of the gaps between the elements of $\mathcal{M}(\alpha, \mathcal{N})$ are continuous functions of $\alpha$. Thus there is an $\varepsilon=\varepsilon(\alpha, \mathcal{N})>0$ such that

$$
l(\beta, \mathcal{N}) \geq l(\alpha, \mathcal{N})
$$

for any $\beta \in(\alpha-\varepsilon, \alpha+\varepsilon)$. If $\alpha$ and $\mathcal{N}$ are chosen as above such that $l(\alpha, \mathcal{N})$ is largest, then one has equality in (1). Replacing if necessary $\alpha$ by an irrational number $\beta \in(\alpha-\varepsilon, \alpha+\varepsilon)$ we may assume in the following that $0<\alpha<1$ is irrational. This last assumption is not essential in our proof, but it makes the presentation cleaner. For instance, in this case the fractional parts $\{n \alpha\}, n \in \mathcal{N}$, will be distinct, and we will discuss in detail the order of these fractional parts. To proceed, recall Dirichlet's theorem which asserts that for any positive integer $M$ there are coprime integers $a, q$ with $1 \leq q \leq M$ such that

$$
\left|\alpha-\frac{a}{q}\right|<\frac{1}{q M} \text {. }
$$

We use (2) with $M=2 N$, so let $a \in \mathbb{Z}$ and $1 \leq q \leq 2 N$ such that $(a, q)=1$ and

$$
\left|\alpha-\frac{a}{q}\right|<\frac{1}{2 q N} \text {. }
$$

Since $0<\alpha<1$ we see that $0 \leq a \leq q$. From (3i) it follows that for any $n \in \mathcal{N}$ one has

$$
\left|n \alpha-\frac{n a}{q}\right|<\frac{1}{2 q} .
$$

Let us consider the open intervals $J_{k}=\left(\frac{k}{q}-\frac{1}{2 q}, \frac{k}{q}+\frac{1}{2 q}\right), k=0,1, \ldots, q-1$. For any $n \in \mathcal{N}$ let $k(n) \in\{0,1, \ldots, q-1\}$ be such that $a n \equiv k(n)(\bmod q)$. Then 
the fractional part $\left\{\frac{a n}{q}\right\}$ coincides with the center $\frac{k(n)}{q}$ of the interval $J_{k(n)}$, and from (44) it follows that $\{n \alpha\}$ belongs to $J_{k(n)}$. Therefore for any $n, n^{\prime} \in \mathcal{N}$ for which $k(n) \neq k\left(n^{\prime}\right)$ the order of the elements $\{n \alpha\},\left\{n^{\prime} \alpha\right\} \in \mathcal{M}$ will simply be given by the order of the numbers $k(n)$ and $k\left(n^{\prime}\right)$. On the other hand, if $n, n^{\prime} \in \mathcal{N}$ are such that $k(n)=k\left(n^{\prime}\right)$, then the order of $\{n \alpha\},\left\{n^{\prime} \alpha\right\}$ is determined by the sign of $\alpha-\frac{a}{q}$ and the order of the numbers $n$ and $n^{\prime}$. To be precise, let $\alpha-\frac{a}{q}=\eta$ and assume in what follows that $\eta>0$. The case $\eta<0$ is similar and will be left to the reader. Since $n \alpha=n \eta+\frac{n a}{q}$, where as we know $|n \eta|<\frac{1}{2 q}$, the relative "coordinate" of $\{n \alpha\}$ with respect to the center $\frac{k(n)}{q}$ of $J_{k(n)}$ will equal $n \eta$. With our assumption on $\eta$, the order of $\{n \alpha\},\left\{n^{\prime} \alpha\right\}$ in case $k(n)=k\left(n^{\prime}\right)$ will be the same as the order of $n, n^{\prime}$. Here the condition $k(n)=k\left(n^{\prime}\right)$ is equivalent to the condition $n \equiv n^{\prime}(\bmod q)$. We now have a more clear picture of the distribution of the elements of $\mathcal{M}$. Write $\mathcal{N}=\bigcup_{r=0}^{q-1} \mathcal{N}_{r}$, where $\mathcal{N}_{r}=\{n \in \mathcal{N}: n \equiv r(\bmod q)\}$. Each $\mathcal{N}_{r}$ corresponds uniquely to a $J_{k}$, given by $k=k(r) \equiv \operatorname{ar}(\bmod q)$, respectively $r=r(k) \equiv \bar{a} k(\bmod q)$, where $\bar{a}$ denotes the inverse of $a \bmod q$. For any $r$, the map $n \mapsto\{n \alpha\}$ sends $\mathcal{N}_{r}$ monotonically to a subset of $J_{k(r)}$. We now distinguish two kinds of gaps $\left(\{n \alpha\},\left\{n^{\prime} \alpha\right\}\right)$ between consecutive elements $\{n \alpha\},\left\{n^{\prime} \alpha\right\}$ of $\mathcal{M}$, according as to whether $k(n)=k\left(n^{\prime}\right)$ or $k(n) \neq k\left(n^{\prime}\right)$, and count them separately. Denote by $l_{1}$, respectively $l_{2}$, the number of distinct lengths of gaps of the first kind, respectively of the second kind, between consecutive elements of $\mathcal{M}$. Some gaps of the first kind might have the same lengths as certain gaps of the second kind. Anyway one has

$$
l(\alpha, \mathcal{N}) \leq l_{1}+l_{2}
$$

In order to get an upper bound for $l_{1}$, we allow $r$ to run over the set $\{0,1, \ldots, q-1\}$ and for each such value of $r$ we look at the gaps formed by the image of $\mathcal{N}_{r}$ in $J_{n(r)}$. We already know that consecutive elements of $\mathcal{N}_{r}$ correspond to consecutive elements of $\mathcal{M}$. Moreover, if $n<n^{\prime}$ are consecutive elements of $\mathcal{N}_{r}$, then the length of the gap between $\{n \alpha\}$ and $\left\{n^{\prime} \alpha\right\}$ equals the difference between their coordinates in $J_{k(r)}$, which is $\left(n^{\prime}-n\right) \eta$. Thus the lengths of these gaps in $\mathcal{M}$ are proportional to the lengths of the gaps $\left(n^{\prime}-n\right)$ in $\mathcal{N}_{r}$, by a factor $\eta$ which is independent of $r$. It follows that $l_{1}$ equals the cardinality of the set

$$
A=\bigcup_{r=0}^{q-1}\left\{n^{\prime}-n: n, n^{\prime} \text { consecutive in } \mathcal{N}_{r}\right\}
$$

Now the point is that since each element of $A$ is a positive multiple of $q$, the sum of its $l_{1}$ (distinct) elements will be at least

$$
q+2 q+\cdots+l_{1} q=\frac{q l_{1}\left(l_{1}+1\right)}{2} .
$$

On the other hand, if we add all the elements of $A$ counted with multiplicities, the sum will equal

$$
\sum_{r=0}^{q-1} \sum_{n, n^{\prime}}\left(n^{\prime}-n\right)=\sum_{r=0}^{q-1}\left(\max \mathcal{N}_{r}-\min \mathcal{N}_{r}\right)<N q .
$$

It follows that $\frac{q l_{1}\left(l_{1}+1\right)}{2}<N q$, which implies

$$
l_{1}<\sqrt{2 N} \text {. }
$$


We now turn to $l_{2}$. Some of the above sets $\mathcal{N}_{r}$ might be empty, resulting in some intervals $J_{k}$ having no points from $\mathcal{M}$. Let $0 \leq k_{1}<k_{2}<\cdots<k_{s} \leq q-1$ be those values of $k$ for which $J_{k} \cap \mathcal{M}$ is nonempty. Then for each pair $\left(k_{j}, k_{j+1}\right)$ we have exactly one gap of the second kind. Its left and right endpoints are the largest element of $\mathcal{M} \cap J_{k_{j}}$ and, respectively, the smallest element of $\mathcal{M} \cap J_{k_{j+1}}$. Thus the length of this gap, which we denote by $\delta_{j}$, is given by

$$
\delta_{j}=\left\{\underline{n}_{j+1} \alpha\right\}-\left\{\bar{n}_{j} \alpha\right\},
$$

where for any $j, \underline{n}_{j}$ and $\bar{n}_{j}$ stand for the smallest, respectively the largest, element of $\mathcal{N}_{r\left(k_{j}\right)}$. The distance between the centers of $J_{k_{j}}$ and $J_{k_{j+1}}$ equals $\frac{k_{j+1}-k_{j}}{q}$ and the coordinates of $\left\{\bar{n}_{j} \alpha\right\}$ and $\left\{\underline{n}_{j+1} \alpha\right\}$ with respect to these centers are $\bar{n}_{j} \eta$ and respectively $\underline{n}_{j+1} \eta$. Hence

$$
\delta_{j}=\frac{k_{j+1}-k_{j}}{q}+\underline{n}_{j+1} \eta-\bar{n}_{j} \eta .
$$

A trivial upper bound for $l_{2}$ is

$$
l_{2} \leq s \leq q .
$$

For each positive integer $b$, let $n(b)$ be the number of distinct lengths $\delta_{j}$ of gaps of the second kind for which $k_{j+1}-k_{j}=b$. Thus $l_{2}$ can be written as

$$
l_{2}=\sum_{b \geq 1} n(b) .
$$

Here we used the fact that if $k_{j+1}-k_{j}=b \neq b^{\prime}=k_{j^{\prime}+1}-k_{j^{\prime}}$, then $\delta_{j} \neq \delta_{j^{\prime}}$. This is a consequence of the inequalities $k_{j+1}-k_{j}-\frac{1}{2}<q \delta_{j}<k_{j+1}-k_{j}+\frac{1}{2}$, which in turn follow from (7) and the inequality $0 \leq n \eta<\frac{1}{2 q}$, valid for any $n \in \mathcal{N}$. Note that

$$
\sum_{b \geq 1} n(b) b \leq \sum_{j}\left(k_{j+1}-k_{j}\right) \leq q .
$$

We claim that for any $b$ one has

$$
n(b) \leq\left[\frac{2 N}{q}\right]+1
$$

where $[\cdot]$ denotes the greatest integer part function. In order to prove the claim, let $j_{1}, \ldots, j_{n(b)}$ be such that $\delta_{j_{1}}, \ldots, \delta_{j_{n(b)}}$ are distinct and

$$
k_{j_{1}+1}-k_{j_{1}}=\cdots=k_{j_{n(b)}+1}-k_{j_{n(b)}}=b .
$$

By (7) we know that

$$
\delta_{j}=\frac{b}{q}+\eta\left(\underline{n}_{j+1}-\bar{n}_{j}\right)
$$

for any $j \in\left\{j_{1}, \ldots, j_{n(b)}\right\}$. The numbers $\delta_{j_{1}}, \ldots, \delta_{j_{n(b)}}$ being distinct, it follows that as $j$ runs over the set $\left\{j_{1}, \ldots, j_{n(b)}\right\}$, the numbers $\underline{n}_{j+1}-\bar{n}_{j}$ are distinct. Recall that $\bar{n}_{j} \in \mathcal{N}_{r\left(k_{j}\right)}$ and $\underline{n}_{j+1} \in \mathcal{N}_{r\left(k_{j+1}\right)}$, so they satisfy the congruences

$$
\bar{n}_{j} \equiv r\left(k_{j}\right) \equiv \bar{a} k_{j}(\bmod q)
$$

and

$$
\underline{n}_{j+1} \equiv r\left(k_{j+1}\right) \equiv \bar{a} k_{j+1}(\bmod q) \text {. }
$$


Hence

$$
\underline{n}_{j+1}-\bar{n}_{j} \equiv \bar{a}\left(k_{j+1}-k_{j}\right) \equiv \bar{a} b(\bmod q)
$$

for any $j \in\left\{j_{1}, \ldots, j_{n(b)}\right\}$. Note also that for any $j$ one has

$$
-N \leq \underline{n}_{j+1}-\bar{n}_{j} \leq N .
$$

There are at most $1+\left[\frac{2 N}{q}\right]$ integers in the interval $[-N, N]$ which are congruent to $\bar{a} b \bmod q$, and this proves (11). Next, from (9) we know that the left-hand side of (10) is a sum of exactly $l_{2}$ terms, counting with multiplicities. By using (11) one sees that the left-hand side of (10) is at least as large as the sum

$$
\left(1+\left[\frac{2 N}{q}\right]\right) \cdot 1+\left(1+\left[\frac{2 N}{q}\right]\right) \cdot 2+\cdots+\left(1+\left[\frac{2 N}{q}\right]\right) u+v(u+1),
$$

where $u$ and $v$ are given by

$$
u=\left[\frac{l_{2}}{1+[2 N / q]}\right]
$$

and

$$
v=l_{2}-\left(1+\left[\frac{2 N}{q}\right]\right) u
$$

We combine this with (10) to derive

$$
\left(1+\left[\frac{2 N}{q}\right]\right) \frac{u(u+1)}{2} \leq q
$$

which implies

$$
u<\left(\frac{2 q}{1+[2 N / q]}\right)^{\frac{1}{2}}
$$

Relations (12) and (13) give

$$
\frac{l_{2}}{1+[2 N / q]}-1<\left(\frac{2 q}{1+[2 N / q]}\right)^{\frac{1}{2}},
$$

from which we get the following upper bound for $l_{2}$ :

$$
l_{2}<1+\left[\frac{2 N}{q}\right]+\left(2 q\left(1+\left[\frac{2 N}{q}\right]\right)\right)^{\frac{1}{2}}<1+\frac{2 N}{q}+(2 q+4 N)^{\frac{1}{2}} .
$$

Since $q \leq 2 N$, from (14) we obtain

$$
l_{2}<1+\frac{2 N}{q}+2 \sqrt{2 N} .
$$

This inequality is sharp when $q$ is at least of the size of $\sqrt{N}$. If $q$ is smaller, then we use (8). From (8) and (15) we find that

$$
l_{2}<(2+\sqrt{2}) \sqrt{N}
$$

regardless of the size of $q$. On combining (5), (6) and (16) we get

$$
l(\alpha, \mathcal{N})<(2+2 \sqrt{2}) \sqrt{N},
$$

which completes the proof of Theorem 1 . 


\section{REFERENCES}

[1] BAKER R.C., Diophantine inequalities, London Math. Soc. Monographs. New Series, 1. The Clarendon Press, Oxford Univ. Press, New York, 1986. MR 88f:11021

[2] BOCA F. and ZAHARESCU A., Pair correlation of values of rational functions $(\bmod p)$, Duke Math. Journal 105 (2000), no 2, 267-307. MR 2001j:11065

[3] MARKLOF, J., The n-point correlations between values of a linear form. With an appendix by Zeev Rudnick, Ergodic Theory Dynam. Systems 20 (2000), no. 4, 1127-1172. MR 2001m:11112

[4] van RAVENSTEIN, T., The three gap theorem (Steinhaus conjecture), J. Austral. Math. Soc. (Series A) 45 (1988), no. 3, 360-370. MR 90a:11076

[5] RUDNICK Z. and SARNAK P., The pair correlation function of fractional parts of polynomials, Comm. Math. Phys. 194 (1998), 61-70. MR 99g:11088

[6] RUDNICK Z., SARNAK P. and ZAHARESCU A., The distribution of spacings between the fractional parts of $n^{2} \alpha$, Invent. Math. 145 (2001), no 1, 37-57. MR 2002e:11093

[7] RUDNICK Z. and ZAHARESCU A., A metric result on the pair correlation of fractional parts of sequences, Acta Arith. 89 (1999), no.3, 283-293. MR 2000h:11083

[8] SCHMIDT W.M., Small fractional parts of polynomials, Regional Conference Series in Mathematics, No. 32, Amer. Math. Soc., Providence, RI, 1977. MR 56:15568

[9] SÓS, V. T., On the distribution mod 1 of the sequence no, Ann. Univ. Sci. Budapest, Eötvös Sect. Math. 1 (1958), 127-134.

[10] ŚWIERCZKOWSKI, S., On successive settings of an arc on the circumference of a circle, Fund. Math. 46 (1958), 187-189. MR 21:3404]

[11] WEYL, H., Uber die Gleichverteilung von Zahlen mod. Eins. Math. Ann. 77 (1916 ), 313-352.

Institute of Mathematics of the Romanian Academy, P.O. Box 1-764, Bucharest 70700, ROMANIA

E-mail address: mvajaitu@stoilow.imar.ro

Institute of Mathematics of the Romanian Academy, P.O. Box 1-764, Bucharest 70700, Romania - and - Department of Mathematics, University of Illinois at UrbanaChampaign, Altgeld Hall, 1409 W. Green Street, Urbana, Illinois 61801

E-mail address: zaharesc@math.uiuc.edu 